\title{
A Mechanically Stable, Low Profile, Omni-Directional Solar-Cell Integrated Antenna for Outdoor Wireless Sensor Nodes
}

\author{
T. Wu, R.L. Li, and M. M. Tentzeris \\ School of Electrical and Computer Engineering \\ Georgia Institute of Technology, Atlanta, GA 30332-0250, U.S.A.
}

\section{Introduction}

For wireless sensor networks, one of the performance bottlenecks is node lifetime [1]. A well designed external interface that incorporates power scavenging, stable mechanics, and efficient wireless transmission can improve node lifetime and would be critical for the massive deployment of wireless networks in outdoor situations, such as environmental control, telematics, structural monitoring and military sensing. To date, several wireless sensor nodes have been integrated with solar cells to increase the robustness of the network [2]. To boost communication range, monopole antenna has been utilized in most nodes because it achieves an omnidirectional radiation pattern desired by ground to ground communication link between the sensor nodes. However, a monopole antenna perpendicular to ground is not mechanically stable, and thus requires additional packaging to avoid node failure from the unexpected outdoor environments. Annular slot loop antennas, ASA, can have similar radiation characteristic to a monopole antenna on a planar surface parallel to ground, thus dramatically reducing the antenna profile. However, since a shorting across the slot would tilt the radiation pattern towards one direction [3], an ASA cannot operate as desired when placed in the same board space under the solar cell circuitry. Several solar antenna integration methods have been published to this date [4]-[5], but all of them are for ground to space applications where a directive antenna is favorable. In this paper, a planar, ground-to-ground, $2.4 \mathrm{GHz}$ solar antenna suitable for 802.15 .4 wireless sensor network standard is proposed by integrating a circular array of slot antennas with solar cells.

\section{Circular Slot Antenna Design}

The "solar-enabled" antenna consists of 4 metal layers as shown in Fig 1. The top three layer thicknesses are given by PCBexpress's 4-layer FR4 $\left(\varepsilon_{\mathrm{r}}=4.6 \sim 4.7\right)$ fabrication standard. FR4 process is chosen because it is a cheap, industrialized process that is ready for immediate massive deployment. The slot antenna layer and the feeding network are shown in Fig 2. Slot antenna is chosen as the radiator because its $\mathrm{E}$ - and $\mathrm{H}$-field are perpendicular and parallel to the ground plane, respectively, when the wave propagates along the ground. [6] This is the same propagation orientation as the $\mathrm{E}$ - and $\mathrm{H}$-field generated by a monopole normal to ground. The two slots on each axis were fed $180^{\circ}$ degrees out of phase to generate an end fire pattern along the ground. The feeding network divides the input signal equally and generates a $180^{\circ}$ degrees out-of-phase signal by exciting the slots in opposite directions. The overall dimensions are shown in Table 1. The structure is simulated and optimized using HFSS. Fig. 3 shows the antenna return loss achieving a $\mathrm{S} 11<-12 \mathrm{~dB}$ from $2.35 \mathrm{GHz}$ to $2.5 \mathrm{GHz}$ with/without the solar cell circuitry, for an elevation of $2 \mathrm{~cm}$ above an infinite earth of $\sigma=0.01 \mathrm{~S} / \mathrm{m}$ and $\varepsilon_{\mathrm{r}}=5$ [6]. The radiation pattern, shown in Fig. 4, includes the simulated radiation 
performance in free space as well as above an infinite ground. The difference in the $\theta=90^{\circ}$ directivity for the $\mathrm{x}$ - and $\mathrm{y}$ - cut is mainly caused by the size difference in GW and GL. These simulation results show that the proposed antenna covers the $2.4 \mathrm{GHz}$ to $2.5 \mathrm{GHz}$ ISM band, and most of the radiated energy is radiated away from the zenith. The antenna is currently submitted for fabrication and the measurement results will be available at the conference.

Table 1. Dimensions of the Circular Slot Antenna

\begin{tabular}{|l|l|l|l|l|l|}
\hline h1 & $12 \mathrm{mils}$ & fP & 10.8 & sL_X1,X2,Y2 & $32.9 \mathrm{~mm}$ \\
\hline h2 & $40 \mathrm{mils}$ & fO (all) & $3 \mathrm{~mm}$ & sL_Y1 & $31.7 \mathrm{~mm}$ \\
\hline h3 & $5 \mathrm{~mm}$ & fS (all) & $4.5 \mathrm{~mm}$ & & \\
\hline GL & $150 \mathrm{~mm}$ & sL_Y1 & $3.8 \mathrm{~mm}$ & & \\
\hline GW & $100 \mathrm{~mm}$ & sL_X1,X2,Y2 & $3 \mathrm{~mm}$ & & \\
\hline
\end{tabular}

The bottom ground layer is critical in stabilizing the matching bandwidth of the slot antenna and providing ground isolation for the signal line. Fig. 6 shows how the bandwidth of a single slot changes with the ground spacing $h_{3}$. The thickness of $h_{3}=5 \mathrm{~mm}$ was chosen based on achieved bandwidth, compactness, and material availability.

\section{Solar Cell Circuitry Design and Power Control Circuit}

The top solar cell circuitry shown in Fig 7 is positioned to avoid blocking the radiating slots from the second layer. The structure consists of $1220 \times 40 \mathrm{~cm}$, $0.6 \mathrm{Voc}$, monocrystalline silicon solar cells in series to generate a $7.2 \mathrm{~V}$ open circuit supply. The $20 x 40 \mathrm{~cm}$ cell is obtained by cutting a 5 " wafer, shown in Fig. 5 , with a dicing saw. The maximum power voltage of the structure would be $5.4 \mathrm{~V}$ assuming a worst case $25 \%$ voltage drop [7]. This is capable of charging lithiumion battery at $4.2 \mathrm{~V}$, along with supplying a wireless sensor node power at $3 \mathrm{~V}$. A power management circuit based on [8] has been built with TI bq24031 and LTC1771. The circuit supplies voltage between 4.2 to $6 \mathrm{~V}$ to charge the battery at sleep mode, and discharges the battery to supply the wireless sensor node at low supply conditions. A video demo of this circuit will be available at the conference. Combining these with the sleep and wakeup strategies mentioned in [2], compact autonomous nodes can be achieved.

\section{Conclusion}

A novel mechanically stable, omnidirectional, low profile "solar-enabled" antenna is proposed and features very good radiation and matching characteristics suitable for the $2.4 \mathrm{GHz}$ wireless sensor network protocol. By using cheap, realizable fabrication process to achieve a compact integration, the proposed antenna integration concept could be a disruptive technology that would potentially enable truly autonomous, robust wireless sensor network in "rugged" outdoor environment.

\section{Acknowledgement}

The authors would like to thank Georgia Electronic Design Center for supporting this research. Additional thanks to Dr. Ajeet Rohatgi and Vijay Yelundur of Georgia Tech UCEP for providing the solar cells. The authors would also like to acknowledge Dr. Charles Theurer, Li Zhang, and Joe Salvo of GE Research for the support of the power management circuit. 


\section{References}

[1] Karl, H., and Willig, A., Protocols and Architecture for Wireless Sensor Networks. Chichester: Wiley, 2005.

[2] Niyato, D., Hossain, E., and Fallahi, A., "Sleep and wakeup strategies in solar-powered wireless sensor/mesh networks: performance analysis and optimization," IEEE Transactions on Mobile Computing, vol. 6, issue 2, pp. 221-236, Feb 2007.

[3] Morishita, H., Hirasawa, K, Fujimoto, K.," Analysis of a cavity-backed annular slot antenna with one point shorted" Antennas and Propagation, IEEE Trans. Antennas and Propagation, vol.39, no.10, pp.1472 - 1478, October 1991.

[4] Henze, N., Giere, A., Fruchting, H., Hofmann, P., "GPS patch antenna with photovoltaic solar cells for vehicular applications," IEEE VTC, vol 1, pp.50-54, October 2003.

[5] Huang, J., "Mars rover antenna for solar-array integration," The Telecommunications and Mission Operations Progress Report, TMO PR 42-136, October-December 1998, pp. 1-13.

[6] Balanis, C., Antenna Theory Analysis and Design $2^{\text {nd }}$ Edition, New York: Wiley, 1997.

[7] Wiles, J., "Perspectives on PV," IAEI magazine, August 2004. http://www.iaei.org/subscriber/magazine/04_d/wiles.htm

[8] Nigel Smith, "Dynamic Power Path Management Simplifies Battery Charging from Solar Panels." TI Application Notes, October 2006. http://focus.ti.com/lit/an/slua394/slua394.pdf

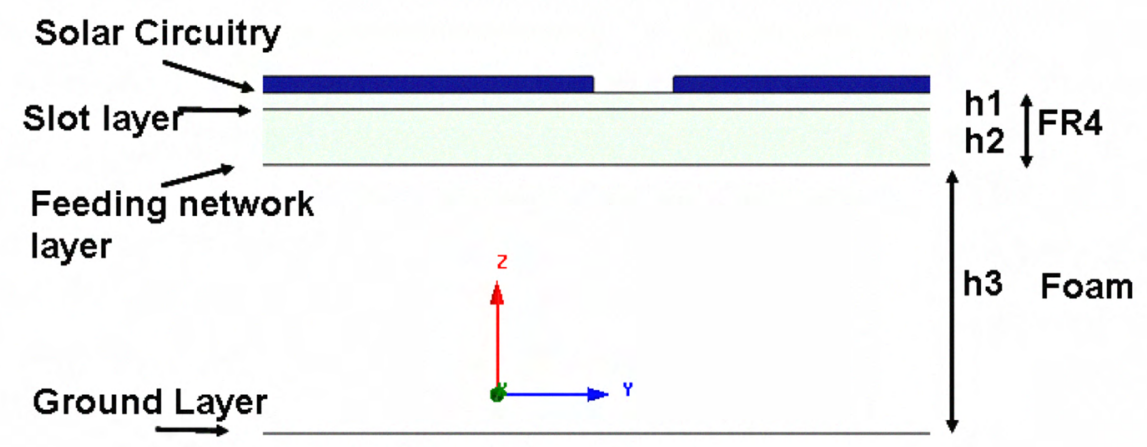

Fig. 1. Solar antenna layers. Metal layer definition on left, substrate layer definitions on right.

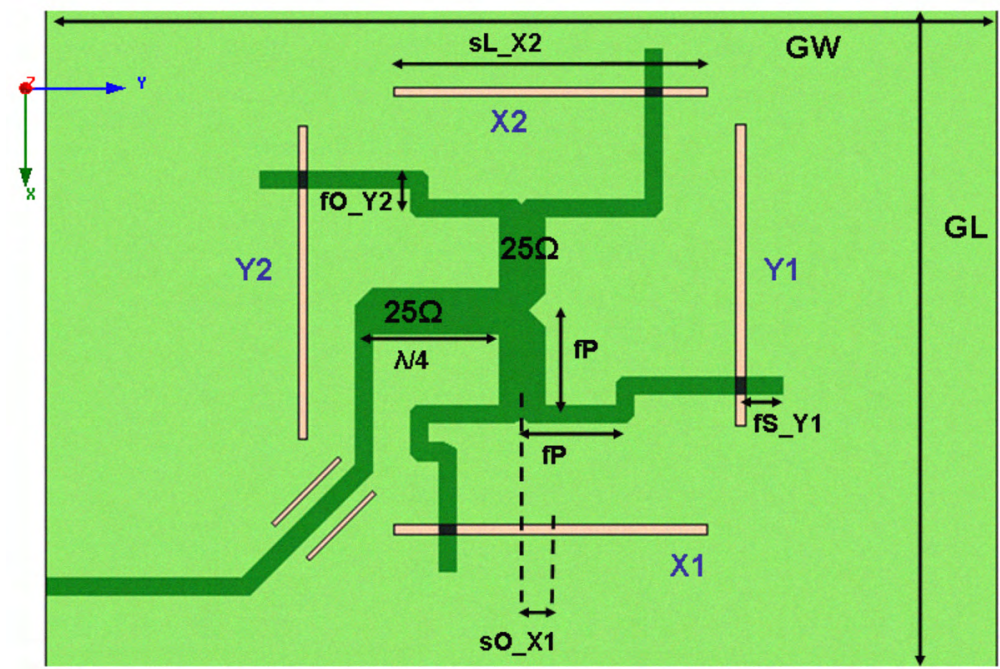

Fig. 2. Slot antenna and feeding network layout (layers 2 and 3) 

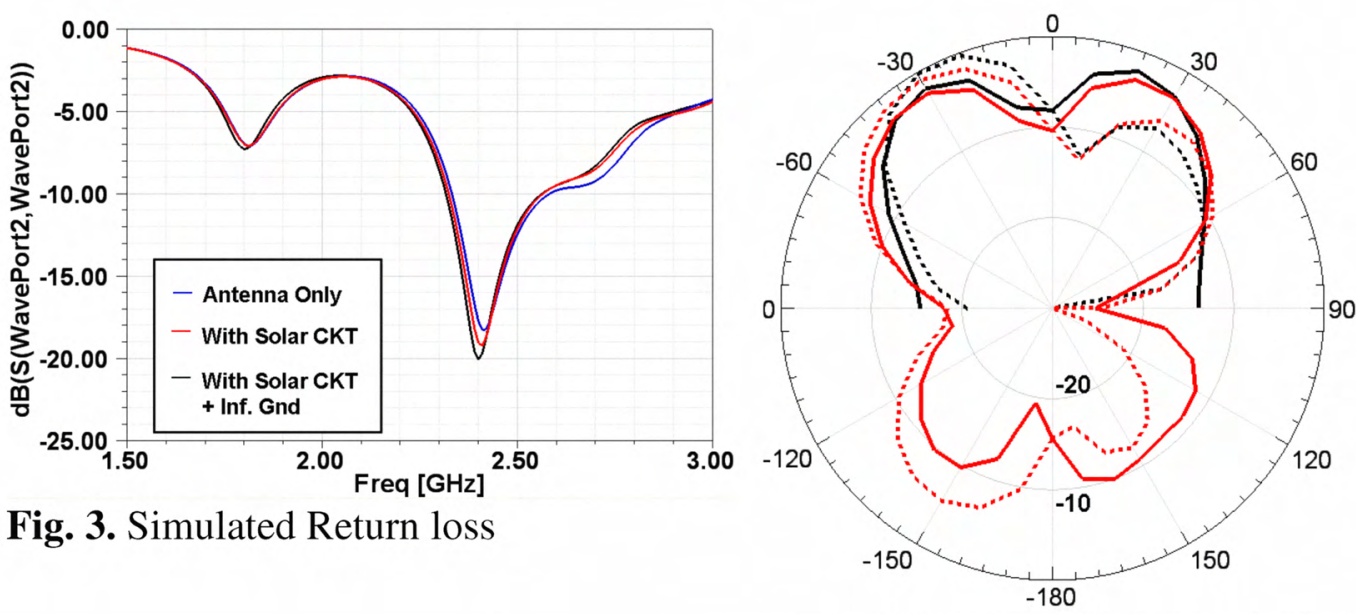

Fig. 3. Simulated Return loss

Fig. 4. $2.4 \mathrm{GHz}$ radiation pattern for free space (red) and infinite "earth" plane (black). Phi $=0$ (solid) $P h i=90$ (dotted)

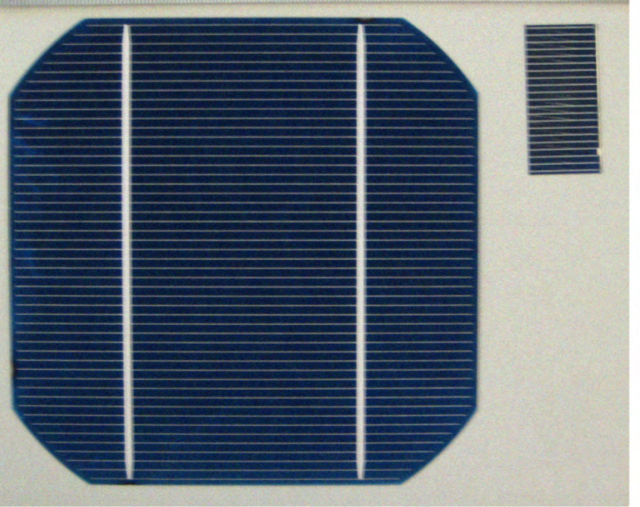

Fig. 5. 5" PV wafer, and $2 \times 4 \mathrm{~cm}$ cut sample cell used for the integration.

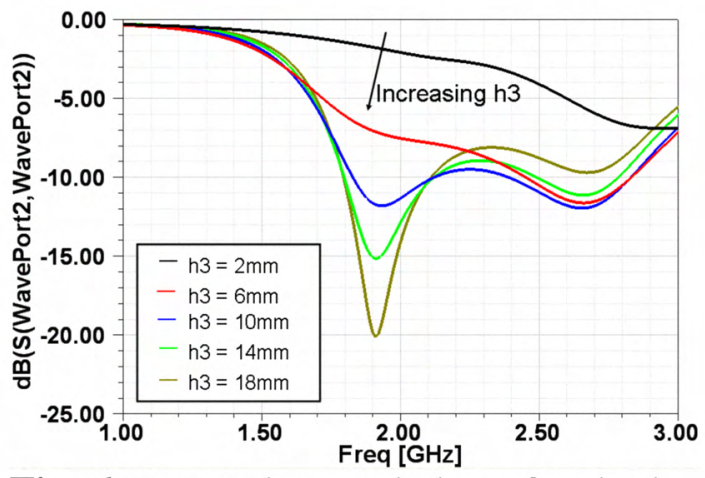

Fig. 6. Return loss variation of a single slot due to h3

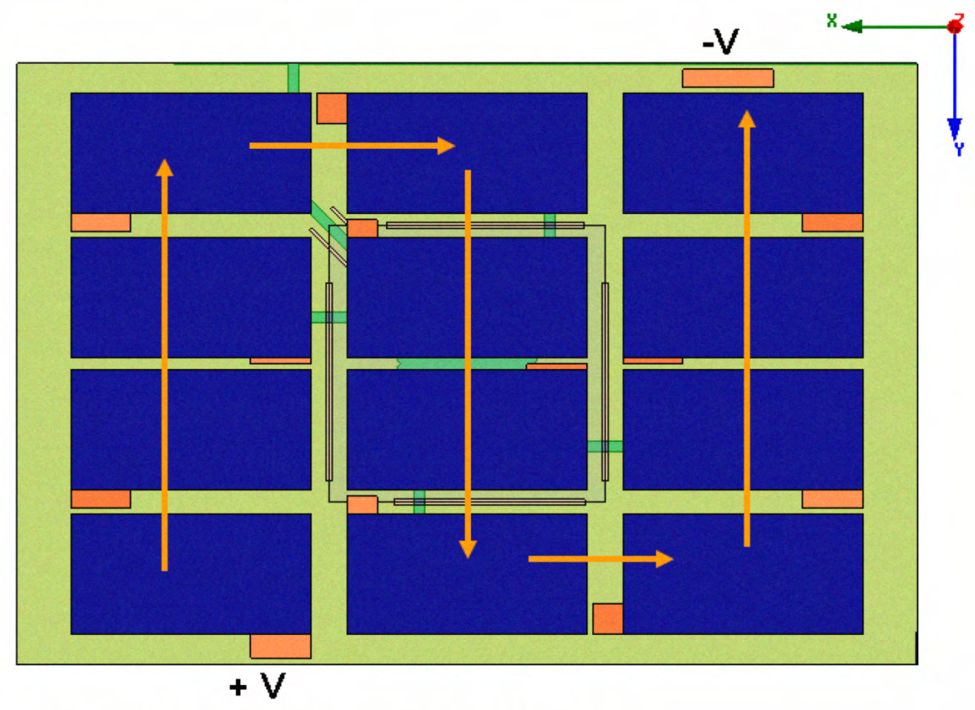

Fig. 7. Solar circuit layout (layer 1) 